\title{
Phase II Trial of Doxorubicin Plus Escalated High-Dose Ifosfamide in Patients With Advanced Soft Tissue Sarcomas of the Adult: A Study of the Spanish Group for Research on Sarcomas (GEIS)
}

\author{
A. López-Pousa, ${ }^{1}$ J. Martín, ${ }^{2}$ J. Montalar, ${ }^{3}$ R. de las Peñas, ${ }^{4} \mathrm{~J}_{\text {. García del Muro, }}{ }^{5} \mathrm{J.} \mathrm{Cruz}^{6}{ }^{6} \mathrm{~J}_{\text {. Maurel, }}{ }^{7}$ \\ P. Escudero, ${ }^{8}$ A. Casado, ${ }^{9}$ J. M. Buesa, ${ }^{10}$ and the Spanish Group for Research on Sarcomas (GEIS) \\ ${ }^{1}$ Medical Oncology Department, Hospital Sant Pau, 08025 Barcelona, Spain \\ ${ }^{2}$ Medical Oncology Department, Hospital Son Dureta, 07014 Palma de Mallorca, Spain \\ ${ }^{3}$ Medical Oncology Department, Hospital Clínico La Fe, 46009 Valencia, Spain \\ ${ }^{4}$ Medical Oncology Department, Hospital Provincial, 12002 Castellón, Spain \\ ${ }^{5}$ Medical Oncology Department, Instituto Catalán de Oncología, 08907 Barcelona, Spain \\ ${ }^{6}$ Medical Oncology Department, Hospital Universitario de Canarias, 38320 Tenerife, Spain \\ ${ }^{7}$ Medical Oncology Department, Hospital Clínic, 08036 Barcelona, Spain \\ ${ }^{8}$ Medical Oncology Department, Hospital Clínico, 50009 Zaragoza, Spain \\ ${ }^{9}$ Medical Oncology Department, Hospital Clínico San Carlos, 28040 Madrid, Spain \\ ${ }^{10}$ Medical Oncology Department, Hospital Central de Asturias, Instituto Universitario de Oncología del Principado \\ de Asturias (IUOPA), 33006 Oviedo, Spain
}

Received 27 December 2005; Revised 22 May 2006; Accepted 6 June 2006

\begin{abstract}
Background. To explore the tolerance and the activity of high-dose ifosfamide (IFOS) combined with doxorubicin (DXR) at 50 $\mathrm{mg} / \mathrm{m}^{2}$ every 4 weeks in patients with soft tissue sarcomas. Methods. DXR was given IV bolus and IFOS by continuous infusion at $2 \mathrm{~g} / \mathrm{m}^{2} /$ day. Initial IFOS dose $\left(12 \mathrm{~g} / \mathrm{m}^{2}\right)$ was adjusted to 10,13 , or $14 \mathrm{~g} / \mathrm{m}^{2}$ according to toxicity. Results. Seventy patients received 277 cycles (median 3 cycles, range 1-10), 34\% with IFOS dose increased, 30\% decreased, and 48\% delivered at $12 \mathrm{~g} / \mathrm{m}^{2}$. Toxicity grade 4 occurred on granulocytes (67\% of patients) or platelets (19\%), $54 \%$ had febrile neutropenia, $31 \%$ grade $3 / 4$ asthenia, and $26 \%$ abandoned the study due to toxicity. Three toxic deaths occurred. In 57 non-GIST patients objective activity was $45.6 \%$ (95\% CI, 32 to $58 \%$ ). Conclusion. At least 4 cycles were tolerated by $71 \%$ of patients, most receiving DXR $50 \mathrm{mg} / \mathrm{m}^{2}$ plus IFOS $10-12$ $\mathrm{g} / \mathrm{m}^{2}$, with substantial toxicity.
\end{abstract}

Copyright (c) 2006 A. López-Pousa et al. This is an open access article distributed under the Creative Commons Attribution License, which permits unrestricted use, distribution, and reproduction in any medium, provided the original work is properly cited.

\section{INTRODUCTION}

Doxorubicin (DXR) and ifosfamide (IFOS) are two active agents against advanced soft tissue sarcomas (ASTS), which have been combined in an attempt to improve therapeutic efficacy [1]. However, the combination of DXR $50 \mathrm{mg} / \mathrm{m}^{2}$ plus IFOS $5 \mathrm{~g} / \mathrm{m}^{2}$ was not more active than single agent DXR at $75 \mathrm{mg} / \mathrm{m}^{2}$ in an EORTC study [2], although other authors have reported a higher response rate when $7.5 \mathrm{~g} / \mathrm{m}^{2}$ IFOS was added to DXR [3] or to a combination of DXR and dacarbazine [4]. The objective activity detected for those IFOS plus DXR combinations ranged from $25 \%$ to $34 \%$. The concourse of hematopoietic growth factors allowed the administration of full DXR doses, but a comparative study of IFOS at $5 \mathrm{~g} / \mathrm{m}^{2}$, combined with 50 or $75 \mathrm{mg} / \mathrm{m}^{2}$ DXR, was negative with respect to efficacy and overall survival, with progression-free survival being significantly longer in the high-dose arm [5].

Second-line studies conducted at higher IFOS doses disclosed a dose-response relationship for this agent $[6,7]$, what originated a series of single-arm Phase II trials combining an anthracycline with high-dose IFOS $\left(\geq 10 \mathrm{~g} / \mathrm{m}^{2}\right)$. At the MD Anderson Hospital Cancer Center, two consecutive trials explored DXR doses of 75 or $90 \mathrm{mg} / \mathrm{m}^{2}$ added to $10 \mathrm{~g} / \mathrm{m}^{2}$ IFOS. All patients included had at least one episode of febrile neutropenia, and thrombocytopenia was significant in both pilot trials, so that $50 \%$ of patients had toxicity grade 4 on 
platelets [8]. When IFOS $12.5 \mathrm{~g} / \mathrm{m}^{2}$ was combined with DXR at $60 \mathrm{mg} / \mathrm{m}^{2}$, grade 4 thrombocytopenia occurred in $13 \%$ of patients, and $74 \%$ received 4 to 6 cycles at full doses [9]. In another study, the combination of $12.5 \mathrm{~g} / \mathrm{m}^{2}$ IFOS with 4epidoxorubicin (EPI) at $90 \mathrm{mg} / \mathrm{m}^{2}$ induced grade 4 thrombocytopenia in $35 \%$ of patients [10], while EPI at $110 \mathrm{mg} / \mathrm{m}^{2}$ plus IFOS $10 \mathrm{~g} / \mathrm{m}^{2}$ were the doses recommended after a Phase I trial [11]. In those trials, grade 4 neutropenia occurred in $70-100 \%$ of patients, a toxicity that was not considered limiting. Objective activity of those regimens, usually delivered every 3 weeks, varied from $52 \%$ to $69 \%$, although randomized studies comparing their efficacy with that of single agent DXR or standard-dose combinations are still lacking.

When this study was planned it appeared that, in those high-dose regimens, the dose of one of the agents should be compromised in order to cause a toxicity that would allow the administration of at least 4 to 6 cycles. Furthermore, our group had recently concluded a first-line trial with IFOS given at $14 \mathrm{~g} / \mathrm{m}^{2}$ every 4 weeks [12]. With those antecedents, we decided to conduct a study to determine the dose of IFOS that would cause an acceptable toxicity when combined with $50 \mathrm{mg} / \mathrm{m}^{2}$ DXR. The initial dose of $12 \mathrm{~g} / \mathrm{m}^{2}$ IFOS was adjusted in subsequent cycles in function of the hematologic toxicity encountered in each patient. Our purpose was also to evaluate the activity of the combination.

\section{MATERIALS AND METHODS}

\section{Selection criteria}

The criteria for inclusion in the trial were a histologic diagnosis of soft tissue sarcoma (malignant mesothelioma and extraosseous chondrosarcoma, osteosarcoma or Ewing/PNET were excluded), locally advanced or metastatic disease, age between 15 and 65 years, no prior chemotherapy either adjuvant or for advanced disease, bidimensionally measurable disease progressing in the last 4 weeks, performance status $(\mathrm{WHO}) \leq 2$, leukocytes $>4.000 / \mathrm{mm}^{3}$, platelets $>100.000 / \mathrm{mm}^{3}$, serum creatinine $<1.5 \mathrm{mg} / \mathrm{dL}$, creatinine clearance $>60 \mathrm{~mL} / \mathrm{min}$, total bilirubin $>1.5 \mathrm{mg} / \mathrm{dL}$, and albumin $>30 \mathrm{~g} / \mathrm{L}$. Patients had to have a left ventricle ejectional fraction (LVEF) at baseline $>50 \%$. Patients were excluded if they had a severe associated disease, CNS metastases, a second primary tumor (except for adequately treated in situ cervical carcinoma and squamous or carcinoma of the skin), if they had received radiation therapy on the only measurable lesion, or if difficulties in follow-up were expected. The study protocol was approved by the Ethical Committee of each participating institution and an informed written consent was obtained from all patients.

\section{Treatment}

All patients received doxorubicin at a fixed dose of $50 \mathrm{mg} / \mathrm{m}^{2}$ infused IV over 10-20 minutes on day 1 immediately followed by a continuous infusion of ifosfamide (IFOS) with GM-CSF support, every 4 weeks. The initial dose of IFOS was $12 \mathrm{~g} / \mathrm{m}^{2}$ (plus 50\% Mesna) (Pras-Pharma, Madrid, Spain) and was given according to the following schedule: on day $1500 \mathrm{~mL}$ of normal saline (NS) were administered IV in 1 hour, followed by IFOS $2 \mathrm{~g} / \mathrm{m}^{2}$ plus Mesna $1 \mathrm{~g} / \mathrm{m}^{2}$ in $1.000 \mathrm{~mL}$ NS in 2 hours, and the rest of the dose was infused at a rate of $2 \mathrm{~g} / \mathrm{m}^{2}$ IFOS every 24 hours. Daily dose was diluted in $1.000 \mathrm{~mL}$ NS. At the end of IFOS infusion 3 additional doses of Mesna $600 \mathrm{mg} / \mathrm{m}^{2}$ every 6 hours were given, either orally or IV, with an oral intake of $1.500 \mathrm{~mL}$ or $1.500 \mathrm{~mL}$ NS IV in 12 hours. All patients received sodium bicarbonate $1 / 6 \mathrm{M} 250 \mathrm{~mL} /$ day (40 mEq/day) with IFOS infusion. No extra fluid intake was required during therapy, and patients were instructed to void frequently. If they noted dysuria, an additional dose of Mesna $1 \mathrm{~g}$ IV bolus was administered and the amount of Mesna in the rest of the infusion was increased to a $100 \%$ that of IFOS. Prophylactic GM-CSF (molgramostin, Schering-Plough SA, Barcelona, Spain) was started on day 7 and given subcutaneously at $5 \mu \mathrm{g} / \mathrm{Kg} /$ day for 10 consecutive days, or until leukocyte count $\geq 10.000 / \mathrm{mm}^{3}$. Values of hemoglobin were kept above $10 \mathrm{~g} / 100 \mathrm{~mL}$ transfusing red-packed cells when necessary.

Weekly BCC were performed, and cycles were repeated if leukocytes were $\geq 3.000 / \mathrm{mm}^{3}$, granulocytes were $\geq$ $1.500 / \mathrm{mm}^{3}$, and platelets were $\geq 100.000 / \mathrm{mm}^{3}$ on day 28 . Otherwise, cycles were delayed until reaching those figures, and the patient interrupted therapy if no recovery was detected after 3 weeks. There were four dose levels of IFOS in this study $\left(10,12,13\right.$, and $\left.14 \mathrm{~g} / \mathrm{m}^{2}\right)$, while the dose of doxorubicin was kept constant at $50 \mathrm{mg} / \mathrm{m}^{2}$. IFOS dose was adapted to the toxicity observed in the prior cycle with the duration of the infusion modified to maintain the rate of $2 \mathrm{~g} / \mathrm{m}^{2} / 24 \mathrm{~h}$. The dose was progressively increased to 13 and $14 \mathrm{~g} / \mathrm{m}^{2}$ if at nadir leukocytes were $\geq 1.000 / \mathrm{mm}^{3}$ and platelets were $\geq 75.000 / \mathrm{mm}^{3}$ in the absence of fever. The dose was reduced one level when neutropenic fever was observed, if platelets were $<50.000 / \mathrm{mm}^{3}$ for more than 1 week, if platelets were $<25.000 / \mathrm{mm}^{3}$, or if stomatitis grade 3 to 4 was reported. If any of those situations recurred at $10 \mathrm{~g} / \mathrm{m}^{2}$, the patient was to abandon the trial. The patient was also withdrawn from the study if creatinine clearance was $<60 \mathrm{~mL} / \mathrm{L}$ or serum creatinine was $>2.5$ times than normal upper value, if a decrease in LVEF > 15\% with respect to baseline was detected, or after any toxicity that might endanger the life of the patient. In the presence of transient hallucinations the patient continued on study, but no if important somnolence, confusion, toxic psychosis or coma (grade 3 to 4 neurocortical toxicity) occurred.

Antiemesis consisted of dexamethasone $8 \mathrm{mg}$ IV bolus plus either granisetron $3 \mathrm{mg}$ or ondansetron $8 \mathrm{mg}$ IV on day 1 , according to each center's routine, followed by oral granisetron $1 \mathrm{mg}$ or ondansetron $4 \mathrm{mg}$ every 12 hours for 6 consecutive days. The use of psychotropic drugs such as benzodiazepines and narcotic analgesics was discouraged during the infusion of IFOS, as well as the use of aminoglycosides to treat febrile episodes.

Baseline studies included a clinical history and physical examination, evaluation of the performance status, assessment of the different tumoral lesions and measurement 
of all evaluable lesions (physical examination, thorax X-ray, computed tomographic scan, but not ultrasound, were valid procedures), thorax X-ray, ECG, LVEF, complete and differential cell blood count (CBC), serum chemistries with glucose, sodium, potassium, calcium, magnesium, phosphorus, bicarbonate, BUN, creatinine, total protein, albumin, AST, ALT, alkaline phosphatase, LDH, total bilirubin, urine examination by microscopy and dipstick, and creatinine clearance. A complete CBC was performed at the end of IFOS infusion and weekly thereafter, while physical examination, evaluation of the performance status, CBC, blood chemistries and microscopic examination of the urine were repeated before each new cycle. A thorax X-ray and the measurement of all evaluable lesions were repeated every 8 weeks, and LVEF was determined every 4 cycles.

WHO criteria were followed to evaluate response to therapy [13]. Any objective remission had to be confirmed at least 4 weeks later and was externally reviewed. Complete remission (CR) was the complete disappearance of all signs and symptoms of disease for at least four weeks, and a pathologic complete response (pCR) was the absence of viable tumor in the surgical specimen of the evaluated lesion. Partial remission (PR) was considered a decrease of at least $50 \%$ in the sum of the products of the two longest perpendicular diameters of every measurable lesion, in the absence of any progressive lesion, and stable disease (SD) was any change that did not qualify for either a partial response or progressive disease. The appearance of a new lesion or an increase of at least $25 \%$ in any measurable lesion was termed progressive disease (PD), while rapid progression was the appearance of a new lesion or an increase in any measurable lesion higher than $50 \%$ after only one cycle of therapy. In the absence of rapid progression, at least 2 cycles were necessary to evaluate antitumor activity. Therapy continued in the presence of a PR or SD and the maximum number of cycles per protocol was 6 , although rescue surgery or other potentially curative procedures could be attempted at the discretion of each investigator only after 4 cycles of therapy for reasons of uniformity. Patients could continue on protocol after $6 \mathrm{cy}$ cles at investigator discretion. All patients receiving at least 1 cycle were considered evaluable for toxicity, and those cycles with weekly CBC were assessable for hematologic toxicity. Toxicity was graded according to Common Toxicity Criteria of the National Cancer Institute (NCI) (Version 1.0). Dose-intensity per patient was calculated by dividing total dose administered per square meter by the total duration of treatment in weeks plus 4 additional weeks.

The duration of objective remissions was measured from the date first observed until the date of progression, and time to progression was measured from the date of inclusion until progressive disease was first observed. In both analysis patients were censored on the date any other therapeutic procedure (ie, rescue surgery) was performed. Overall survival was measured from the date of patient inclusion until death from any cause or last control. Actuarial time to progression and overall survival were determined by the method of Kaplan and Meier. Student- $t$ test was applied to compare mean values. All $p$-values are two-tailed.
Table 1: Patient characteristics.

\begin{tabular}{lc}
\hline Included & 73 \\
Eligible & 71 \\
Male/female & $29 / 42$ \\
Median age (range) & $55(22-65)$ \\
Performance status: & \\
0 & 24 \\
1 & 34 \\
2 & 13 \\
Histology: & \\
Leiomyosarcoma & 16 \\
GIST & 8 \\
Fibrosarcoma & 6 \\
Liposarcoma & 6 \\
Malignant fibrous histiocytoma & 5 \\
Angiosarcoma & 4 \\
Neurosarcoma & 4 \\
Synovial cell sarcoma & 3 \\
Rhabdomyosarcoma & 22 \\
Mixed mesodermal sarcoma & 2 \\
Other & 35 \\
Grade of malignancy: & \\
1 & 2 \\
2 & \\
Sites of disease: & \\
Primary/local recurrence & \\
Lung & \\
\hline
\end{tabular}

To determine the minimum size of the sample necessary to detect at least a $20 \%$ of activity, we followed the two-stage Phase II design of Gehan [14]. Once this activity was confirmed in the first 25 patients, it was decided to continue the study to further characterize the toxicity of the schedule in a multicenter setting.

\section{RESULTS}

\section{Patient characteristics}

From July 1997 to July 1999, 73 patients were included in the study. Two were considered ineligible (one with a low performance status and another exposed to prior chemotherapy). One patient was not evaluable for any analysis due to lack of data, 70 were assessable for toxicity and 64 for antitumor activity. Six patients were not valid for analysis of efficacy: 5 received only 1 cycle and one patient had a toxic death after the second cycle. Main characteristics of eligible patients are presented in Table 1. Retrospectively, a diagnosis of gastrointestinal stromal sarcoma tumor (GIST) was done 
TABLE 2: Number of patients and dose of ifosfamide during the first 6 cycles of treatment.

\begin{tabular}{|c|c|c|c|c|c|}
\hline & \multicolumn{4}{|c|}{ Dose of ifosfamide } & \multirow{3}{*}{$\begin{array}{c}\text { Total } \\
\text { (patients) }\end{array}$} \\
\hline & $10 \mathrm{~g} / \mathrm{m}^{2}$ & $12 \mathrm{~g} / \mathrm{m}^{2}$ & $13 \mathrm{~g} / \mathrm{m}^{2}$ & $14 \mathrm{~g} / \mathrm{m}^{2}$ & \\
\hline Cycle number & & & & & \\
\hline 1 & $5^{\text {(a) }}(0.07)$ & $65(0.93)$ & - & - & 70 \\
\hline 2 & $19(0.30)$ & $23(0.36)$ & $22(0.34)$ & - & 64 \\
\hline 3 & $18(0.37)$ & $17(0.35)$ & $4(0.08)$ & $10(0.20)$ & 49 \\
\hline 4 & $15(0.40)$ & $10(0.27)$ & $4(0.11)$ & $8(0.22)$ & 37 \\
\hline 5 & $9(0.37)$ & $6(0.25)$ & $3(0.13)$ & $6(0.25)$ & 24 \\
\hline 6 & $8(0.40)$ & $6(0.30)$ & $2(0.10)$ & $4(0.20)$ & 20 \\
\hline
\end{tabular}

(a) Number of patients (\%).

in 8 patients. In 20 patients the primary tumor was the only site of disease and the lungs in 16. Sixteen patients had received radiotherapy before.

\section{Treatment}

In total 277 cycles were delivered, with a median of 3 per patient (range 1-10). Six patients received only 1 cycle of therapy due to hematologic [1] or cardiac toxicity [1], treatment refusal [1], rapid progression [1] or a toxic death [2]. According to ifosfamide dose, 81 cycles were given at $10 \mathrm{~g} / \mathrm{m}^{2}, 130$ at $12 \mathrm{~g} / \mathrm{m}^{2}, 33$ at $13 \mathrm{~g} / \mathrm{m}^{2}$, and 34 at $14 \mathrm{~g} / \mathrm{m}^{2}$. In Table 2 we summarized the number of cycles given by ifosfamide dose level during the 6 cycles projected by protocol. Roughly, one third of cycles were given at 10 , at 12 , and at $>12 \mathrm{~g} / \mathrm{m}^{2}$ IFOS during cycles 2 to 6 . Five patients erroneously started therapy at $50 \mathrm{mg} / \mathrm{m}^{2}$ doxorubicin and $10 \mathrm{~g} / \mathrm{m}^{2}$ ifosfamide: 1 refused further therapy, 2 had excessive toxicity for increasing dose, 1 continued at $10 \mathrm{~g} / \mathrm{m}^{2}$ and 1 had the dose of ifosfamide increased to $12 \mathrm{~g} / \mathrm{m}^{2}$ in subsequent cycles. Among the 65 patients who received the first cycle at $12 \mathrm{~g} / \mathrm{m}^{2}$ ifosfamide, 22 had the dose increased to $13 \mathrm{~g} / \mathrm{m}^{2}$ and 16 reduced to $10 \mathrm{~g} / \mathrm{m}^{2}$ in the second cycle. The reasons to abandon the study during the first 6 cycles of therapy are summarized in Table 3. Ten out of 21 patients (48\%) leaving the trial for toxicity were receiving IFOS at $10 \mathrm{~g} / \mathrm{m}^{2}$ and 7 $(33 \%)$ at $12 \mathrm{~g} / \mathrm{m}^{2}$. Twenty patients completed 6 cycles, and 6 of them continued on therapy afterwards. Fifteen percent of the first 6 cycles were delayed, either due to toxicity $(50 \%)$, patient request, or bed availability, median cycle interval being 28 days (range $26-35$ days) with only $5 \%$ of cycles given on day 35 . Mean dose received ( \pm standard deviation) in those cycles was $50 \pm 1.58 \mathrm{mg} / \mathrm{m}^{2}$ for doxorubicin and $11.70 \pm 1.43 \mathrm{~g} / \mathrm{m}^{2}$ for ifosfamide, and mean dose intensity was $11.98 \pm 0.70 \mathrm{mg} / \mathrm{m}^{2} /$ week for DXR and $2.82 \pm 0.34 \mathrm{~g} / \mathrm{m}^{2} /$ week for IFOS.

\section{Hematologic toxicity}

Sixty-nine patients and 254 cycles were assessable for hematologic toxicity. In Table 4 we present the maximum grade of
TABle 3: Reasons to abandon the study during the first 6 cycles of treatment.

\begin{tabular}{|c|c|c|c|c|c|}
\hline & Toxicity ${ }^{(a)}$ & Progression & $\begin{array}{l}\text { Additional } \\
\text { therapy }^{(b)}\end{array}$ & $\begin{array}{l}\text { End of } \\
\text { therapy }\end{array}$ & Total \\
\hline \multicolumn{6}{|l|}{ Cycle number } \\
\hline 1 & $5^{(\mathrm{c})}$ & 1 & - & - & 6 \\
\hline 2 & 8 & 7 & - & - & 15 \\
\hline 3 & 5 & 6 & 1 & - & 12 \\
\hline 4 & 2 & 2 & 5 & 4 & 13 \\
\hline 5 & 1 & 2 & 1 & - & 4 \\
\hline 6 & - & - & 8 & 6 & 14 \\
\hline
\end{tabular}

(a) Toxicity includes excessive toxicity, patient refusals, and toxic deaths.

(b) Surgery, radiotherapy or both.

(c) Number of patients.

this toxicity per patient during the whole treatment period. A grade 3 to 4 decrease in hemoglobin values was observed in $40 \%$ of patients ( $16 \%$ of cycles), and in platelet count in $43 \%$ of patients (19\% of cycles), while $82 \%$ and $79 \%$ had grade 3 to 4 toxicity on leukocytes or granulocytes in $58 \%$ and $58 \%$ of cycles, respectively. The percentage of patients with grades 3 and 4 hematologic toxicity by dose of ifosfamide during the first 6 cycles of therapy is presented in Table 5. In those first 6 cycles, mean platelet values decreased with the number of cycles delivered $(P<.0001)$. In patients with granulocytes $<1.000 / \mathrm{mm}^{3}$ (grade 3 to 4 ), the nadir occurred on day 12 (range 5-30), with mean granulocyte values of $0.22 / \mathrm{mm}^{3}$ (range $0-0.97$ ). In $83 \%$ of cycles, recovery to minimum hematologic values required for therapy was already reached by day 21 .

\section{Nonhematologic toxicity}

The most frequent grade 3 to 4 side-effects were infection ( $46 \%$ of patients), vomiting ( $27 \%)$, asthenia $(31 \%)$, anorexia $(28 \%)$, and stomatitis (10\%) (see Table 6). All episodes of neurocortical toxicity grade 3 consisted of visual hallucinations, while grade 1 to 2 episodes were usually characterized by a mild-to-moderate somnolence. Two patients referred asterixis with limitation to hold objects, and another two noted peripheral paresthesias. Serial LVEF determinations were available from 52 patients, and in 17 a mean decrease of $10.94 \pm 4.14 \%$ was noted after therapy, with only 5 presenting values below 50\% (range $41-47 \%$ ). All 3 patients with serial measurements performed within the first cycle had a transient decrease in LVEF of $17 \%, 20 \%$, and $7 \%$, respectively, with one of them associated with symptomatic left cardiac failure that led to treatment interruption. Another patient who had received a cumulative DXR dose of $250 \mathrm{mg} / \mathrm{m}^{2}$ developed clinical cardiac failure 1 year after the end of protocol therapy, when an LVEF was 30\%. Increased serum creatinine values observed in 9 patients were reversible in 6, 2 with grade 1 toxicity were not further evaluated, and one patient abandoned the study due to persistent creatinine clearance values $<60 \mathrm{~mL} / \mathrm{min}$ concomitantly with a grade 1 
TABle 4: Maximum hematologic toxicity ( $\%$ of patients).

\begin{tabular}{l|rrrrr}
\hline & \multicolumn{5}{|c}{ Grade CTC } \\
\hline & 0 & 1 & 2 & 3 & 4 \\
\hline Hemoglobin & 1 & 6 & 53 & 31 & 9 \\
Leukocytes & 8 & - & 10 & 22 & 60 \\
Granulocytes & 12 & 3 & 6 & 12 & 67 \\
Platelets & 15 & 29 & 13 & 24 & 19 \\
\hline
\end{tabular}

increase in serum creatinine. No episodes of acute renal failure were observed.

During the study 38 patients (20\% of cycles) had at least one episode of febrile neutropenia, requiring 50 hospital admissions for support. Although out-patient treatment through a central catheter was allowed, all patients but one received treatment as inpatients. If days of hospitalization due to both treatment and support measures are added, $75.4 \%$ of cycles required less than 7 days at hospital (median 5, range 3-6 days) and $24.6 \%$ more than 7 days (median 13 , range 8-27 days). Red-packed cell transfusions were given to 41 patients in $29 \%$ of cycles, and 11 received platelet transfusions in 11 cycles. Sixteen patients in 23 cycles referred toxicity to GM-CSF that usually consisted of an itchy erythema plus mild swelling at the site of puncture (13 patients); 3 patients noted night sweats, fever, or a poor tolerance to GMCSF administration. Two patients were shifted to G-CSF. All three episodes of toxic death were secondary to neutropenia plus unrecoverable sepsis in one patient coincidental with neurocortical and renal toxicity.

\section{Response to therapy and evolution}

In 64 patients assessable for activity, the best response observed was a complete remission in 1 patient, a partial remission in 26 , stable disease in 24 , and progressive disease in 13 , for an overall activity rate of $42 \%$ (95\% CI, 30-54\%). In 8 GIST patients, 1 partial remission, 2 stabilizations and 4 progressions were noted, with 1 being nonevaluable. If GIST patients are excluded, response rate was $45.6 \%$ (95\% CI, 32$58 \%)$. Sensitive histotypes were leiomyosarcoma (8/14), angiosarcoma (2/4), neurogenic sarcoma (2/4), rhabdomyosarcoma (1/2), mixed mesodermal sarcoma (1/2), fibrosarcoma (2/6), synovial cell sarcoma (1/3), malignant fibrous histiocytoma (1/4) or liposarcoma (1/5) among others. Activity was noted on the primary tumor (44\%), lung (48\%), liver (33\%), or other lesions $(15 \%)$. Mean time $( \pm \mathrm{SD})$ to achieve an objective remission was $2.8 \pm 1.5$ months (range 1.5-8 months). No correlation was observed between the mean dose of IFOS received and response. Mean duration of response was 7.87 months (95\% CI, 5.88-9.85 months), and mean time to progression was 9 months (95\% CI, 7-11 months).

The reason to abandon the study was excessive toxicity in 16 patients, treatment refusal in 2 (after 1 and 5 cycles, resp), salvage surgery in 6 , disease progression in 19 , and treatment completion in 24. Four patients finished protocol therapy after 4 cycles in the absence of toxicity or progression. Toxicity
TABle 5: Grades 3 and 4 hematologic toxicity by ifosfamide dose during the first 6 cycles ( $\%$ of patients).

\begin{tabular}{l|cccc}
\hline & \multicolumn{4}{|c}{ Dose of ifosfamide } \\
\hline & $10 \mathrm{~g} / \mathrm{m}^{2}$ & $12 \mathrm{~g} / \mathrm{m}^{2}$ & $13 \mathrm{~g} / \mathrm{m}^{2}$ & $14 \mathrm{~g} / \mathrm{m}^{2}$ \\
\hline Hemoglobin & $21 / 7$ & $20 / 3$ & $9 / 4.5$ & $36 / 9$ \\
Leukocytes & $18 / 57$ & $19 / 42$ & $41 / 23$ & $27 / 45$ \\
Granulocytes & $0 / 71$ & $19 / 46$ & $9 / 45$ & $9 / 63$ \\
Platelets & $46 / 11$ & $14 / 9$ & $9 / 9$ & $0 / 36$ \\
\hline
\end{tabular}

that led to treatment interruption consisted of hematologic depression associated to febrile neutropenia [11], poor general tolerance to therapy [4], or a persistent decrease in creatinine clearance $(<60 \mathrm{~mL} / \mathrm{min})$ in 1 patient. The occurrence of excessive toxicity was not related with sex, performance status, or site of disease, but those patients with severe toxicity tended to be older (mean \pm SD, $54 \pm 10$ years versus $49 \pm 9.7$ years, $P=.057$ ). In all, 16 patients were submitted to rescue surgery ( 9 in partial remission and 7 with stable disease) and 11 apparently achieved a complete remission. Five of those patients also received adjuvant radiotherapy, and another 3 patients received radiotherapy upon completion of protocol therapy.

At the time of last analysis, 60 patients (86\%) had died of disease, 3 had a toxic death, 2 were lost to follow-up (one of them without evidence of disease 6 years after completing therapy), and 5 patients were alive, 2 with active disease and 3 with no evidence of disease (two rescued with surgery and one with a durable complete remission after temozolomide). Median ( $\pm \mathrm{ds}$ ) overall survival for the whole series was $17 \pm 2$ months (95\% CI, 13-22 months). No relation between survival and grade of hematologic toxicity was observed.

\section{DISCUSSION}

In this study, ASTS patients were treated with IFOS at an initial dose of $12 \mathrm{~g} / \mathrm{m}^{2}$ combined with DXR $50 \mathrm{mg} / \mathrm{m}^{2}$ every 4 weeks, with the dose of IFOS adjusted in the $10-14 \mathrm{~g} / \mathrm{m}^{2}$ range according to the hematologic nadir. The dose of IFOS was increased in $34 \%$ of patients, and $30 \%$ had the dose reduced to $10 \mathrm{~g} / \mathrm{m}^{2}$ in the second cycle. These proportions were maintained all along the study, so that approximately one third of patients each received 10,12 , and $>12 \mathrm{~g} / \mathrm{m}^{2}$ IFOS (plus $50 \mathrm{mg} / \mathrm{m}^{2} \mathrm{DXR}$ ) during the first 6 cycles, pointing perhaps to interpatient differences in drug metabolism (Tables 2 and 5). A criticism to this trial may be the low dose of DXR administered, which led to a low dose intensity $\left(12.5 \mathrm{mg} / \mathrm{m}^{2} /\right.$ week $)$ compared with the $25 \mathrm{mg} / \mathrm{m}^{2} /$ week of DXR given as a single agent at standard doses. As $83 \%$ of cycles had hematologic values suitable for repeating therapy on day 21 , one can speculate whether this schedule would had been tolerated every 3 weeks. However, $26 \%$ of patients were taken off study due to excessive toxicity and three toxic deaths occurred, indicating that there is little margin for dose increase.

The three studies that explored a combination of IFOS $12 \mathrm{~g} / \mathrm{m}^{2}$ plus DXR $60 \mathrm{mg} / \mathrm{m}^{2}$ repeated every 3 weeks in a 
TABle 6: Nonhematologic toxicity (\% of patients).

\begin{tabular}{l|ccccc}
\hline & \multicolumn{5}{|c}{ Grade } \\
\hline Nausea & 0 & 1 & 2 & 3 & 4 \\
Vomiting & 10.1 & 20.3 & 48 & 20.3 & 1.4 \\
Diarrhea & 18.6 & 17.1 & 37.1 & 24.3 & 2.9 \\
Stomatitis & 81.2 & 11.6 & 5.8 & 1.4 & - \\
Asthenia & 54.3 & 16 & 20 & 8.6 & 1.4 \\
Anorexia & 7.4 & 17.6 & 44.1 & 25 & 6 \\
Cardiotoxicity & 13.2 & 25 & 34 & 23.5 & 4.4 \\
Neurocortical & 81.2 & 11.6 & 3 & 4.3 & - \\
Cutaneous & 55 & 24.6 & 7.2 & 13 & - \\
Alergia & 88 & 6 & 6 & - & - \\
Fever & 93 & 6 & 1 & - & - \\
Infection & 38.6 & 7 & 38.6 & 7.1 & 8.6 \\
Creatinine & 45 & 3 & 6 & 36 & 10 \\
Hematuria & 86 & 11 & 3 & - & - \\
\hline
\end{tabular}

small number of ASTS patients encountered excessive toxicity. In the first, only $85 \%$ of the planned dose for both agents was given [9]. The second was a Phase I trial testing two IFOS dose levels $\left(10\right.$ and $12 \mathrm{~g} / \mathrm{m}^{2}$ ) added to DXR (50$90 \mathrm{mg} / \mathrm{m}^{2}$ ). DXR $60 \mathrm{mg} / \mathrm{m}^{2}$ plus $12 \mathrm{~g} / \mathrm{m}^{2}$ IFOS was the maximum tolerated dose due to the presence of neutropenia lasting more than 4 days and thrombocytopenia grade 4 in 3 out of 4 patients. However, when $10 \mathrm{~g} / \mathrm{m}^{2}$ IFOS were combined with $60-90 \mathrm{mg} / \mathrm{m}^{2}$ DXR, the dose limiting toxicity was not reached [15]. In the third study, 8 out of $14(57 \%)$ patients with metastatic disease treated at DXR $60 \mathrm{mg} / \mathrm{m}^{2}$ plus IFOS $12 \mathrm{~g} / \mathrm{m}^{2}$ every 3 weeks discontinued therapy due to toxicity [16].

In this trial the dose of IFOS was adjusted in each patient in function of the toxicity. The dose limiting toxicity was febrile neutropenia, which affected $54 \%$ of patients, a figure equal to that observed in our Phase II trial with highdose IFOS conducted in a similar patient population [12]. In spite of the prophylactic use of hematopoietic growth factors, grade 4 granulocytopenia occurs in $70-100 \%$ of patients treated with high-dose regimens, and febrile neutropenia is reported in $35-100 \%$ of patients exposed to DXR plus high-dose IFOS $[8,9,15]$ or in $13-54 \%$ of those treated with combinations of EPI plus IFOS at $9-12 \mathrm{~g} / \mathrm{m}^{2}[10,17,18]$, although septic deaths are seldom reported. This grade of toxicity must be accepted as inherent to any high-dose regimen applied to patients with ASTS. Grade 4 thrombocytopenia was detected in $19 \%$ of our patients. It was cumulative in nature, but did not lead to delays or interruptions of therapy. This incidence of grade 4 thrombocytopenia seems acceptable when compared with the $50 \%$ observed in trials which incorporated DXR at $75-90 \mathrm{mg} / \mathrm{m}^{2}$ to $10 \mathrm{~g} / \mathrm{m}^{2}$ IFOS [8], or the $40-75 \%$ of the combination of DXR $60 \mathrm{mg} / \mathrm{m}^{2}$ plus IFOS $12 \mathrm{~g} / \mathrm{m}^{2}[15,16]$, what eventually limits the number of cycles that can be given at full doses. Other toxic effects noted are well known and were similar to those observed by others. In any case, these high-dose schedules are too toxic for routine use and should only be administered in experienced centers.

Due to the toxicity observed in this trial and in order to administer higher doses of DXR, we explored a sequential approach in a subsequent study, administering dose-dense DXR ( 3 cycles at $90 \mathrm{mg} / \mathrm{m}^{2}$ repeated every 2 weeks) followed by high-dose ifosfamide ( 3 cycles at $12.5 \mathrm{~g} / \mathrm{m}^{2}$ every 3 weeks) [19]. In this trial, which included 57 patients, $7 \%$ of patients withdrew due to refusal or excessive toxicity, $24 \%$ had febrile neutropenia, 24\% had thrombocytopenia grade 3 to 4 , and $66 \%$ completed the 6 projected cycles of therapy. This regimen offered a better toxicity profile and compliance when compared with either the single agent IFOS or the presently reported study, with a similar antitumor activity (remission rate $38 \%$; $95 \%$ CI, 25-51\%), and it may provide a therapeutic option for ASTS patients. For this reason, it was selected for comparison with single agent DXR $\left(75 \mathrm{mg} / \mathrm{m}^{2} \mathrm{ev}-\right.$ ery 3 weeks) in a Phase III study, presently under course. Prolongation of IFOS infusion offers another alternative, more cumbersome, to reduce the toxicity of DXR plus high-dose IFOS combinations. The study of the Italian Sarcoma Group showed that IFOS $13 \mathrm{~g} / \mathrm{m}^{2}$ infused over 12 days and bolus DXR at $75 \mathrm{mg} / \mathrm{m}^{2}$ on day 8 could be combined every 4 weeks, with grade 4 neutropenia and thrombocytopenia observed, respectively, in $66 \%$ and $8 \%$ of patients [20].

Antitumor activity in ASTS (excluding GIST patients) was $45.6 \%$, which is below the $54-69 \%$ of figures reported for other high-dose regimens tested $[8,10,15,17,18,20]$, with the exception of the $25 \%$ reported in 12 metastatic patients [16]. Perhaps the low DXR dose administered influenced our results, although this figure coincides with that of other studies from our Group [12, 19], and no definitive conclusions can be drawn from small trials conducted in such a heterogeneous group of tumors. However, the results of most studies are consistent, and suggest that high-dose regimens may have a higher efficacy than standard-dose schedules, an impression that should be tested in adequately sized randomized trials. Salvage surgery was possible in $25 \%$ of evaluable patients, and $56 \%$ of them had obtained a partial remission during protocol therapy. Rescue surgery is performed in about 30\% of ASTS patients included in high-dose studies $[10,17,18]$, being difficult to deduce to what extent chemotherapy has facilitated surgery. For this reason, resectability of lesions should be established beforehand through clear definitions included among the selection criteria. This aspect, addressed in a recent paper [21], could be considered a stratification criterion in future studies.

From the therapeutic results obtained in some solid tumors, it has been proposed that hematologic toxicity is a better parameter than dose intensity to measure dose adequacy, and that outcome is related to the grade of that toxicity [22]. According to this, the doses of any chemotherapeutic regimen should be adjusted to cause at least moderate hematologic toxicity to every patient to compensate for differences in tolerance [23]. In ASTS patients, it is difficult to test that proposal due to the very heterogeneous nature of the tumors grouped under the term sarcoma. With this contention, if toxicity is related to outcome, then the regimen we tested 
would have reached a limit, as well as other high-dose regimens combining IFOS and an anthracycline, that will unlikely be improved by pushing further the doses delivered. New agents would be necessary to improve the poor therapeutic results obtained in ASTS patients, and the differences in biology and in sensitivity between the different sarcoma subtypes should be exploited.

\section{ACKNOWLEDGMENTS}

This work was supported in part by grants from ScheringPlough España SA and Pras-Pharma SA. The study was completed within the framework of Program 9 of the Redes Temáticas de Investigación Cooperativa de Centros de Cáncer (Grant C03/10), Instituto de Salud Carlos III, Ministerio de Sanidad y Consumo, Spain. The Instituto Universitario de Oncología del Principado de Asturias (IUOPA) is supported by Obra Social Cajastur, Asturias, Spain. The authors appreciate the superb data management performed by Dulce Menéndez Cuervo. Also contributed to this work J. Martínez-Trufero MD, Hospital Miguel Servet, Zaragoza; I. Bover MD, Hospital San Joan, Reus, Tarragona; I. Sevilla MD, Hospital Clínico Universitario Virgen de la Victoria, Málaga; C. Balañá MD PhD, Hospital Can Ruti, Badalona; A. Paredes MD, Hospital Ntra Sra de Aranzazu, San Sebastián; J. Carles MD, Hospital del Mar, Barcelona; J. Vicent MD, Hospital General Universitario, Valencia; and J. Cassinello MD, Hospital Universitario, Guadalajara.

\section{REFERENCES}

[1] Brennan MF, Casper ES, Harrison LB. Soft tissue sarcoma. In: DeVita VT, Hellman S, Rosenberg SA, eds. Cancer, Principles and Practice of Oncology. 5th ed. New York, NY: LippincottRaven; 1997:1738-1788.

[2] Santoro A, Tursz T, Mouridsen H, et al. Doxorubicin versus CYVADIC versus doxorubicin plus ifosfamide in first-line treatment of advanced soft tissue sarcomas: a randomized study of the European Organization for Research and Treatment of Cancer Soft Tissue and Bone Sarcoma Group. Journal of Clinical Oncology. 1995;13(7):1537-1545.

[3] Edmonson JH, Ryan LM, Blum RH, et al. Randomized comparison of doxorubicin alone versus ifosfamide plus doxorubicin or mitomycin, doxorubicin, and cisplatin against advanced soft tissue sarcomas. Journal of Clinical Oncology. 1993;11(7):1269-1275.

[4] Antman K, Crowley J, Balcerzak SP, et al. An intergroup phase III randomized study of doxorubicin and dacarbazine with or without ifosfamide and mesna in advanced soft tissue and bone sarcomas. Journal of Clinical Oncology. 1993;11(7):12761285.

[5] Le Cesne A, Judson I, Crowther D, et al. Randomized phase III study comparing conventional-dose doxorubicin plus ifosfamide versus high-dose doxorubicin plus infosfamide plus recombinant human granulocyte-macrophage colonystimulating factor in advanced soft tissue sarcomas: a trial of the European Organization for Research and Treatment of Cancer/Soft Tissue and Bone Sarcoma Group. Journal of Clinical Oncology. 2000;18(14):2676-2684.

[6] Le Cesne A, Antoine E, Spielmann M, et al. High-dose ifosfamide: circumvention of resistance to standard-dose ifos- famide in advanced soft tissue sarcomas. Journal of Clinical Oncology. 1995;13(7):1600-1608.

[7] Palumbo R, Palmeri S, Antimi M, et al. Phase II study of continuous-infusion high-dose ifosfamide in advanced and/or metastatic pretreated soft tissue sarcomas. Annals of Oncology. 1997;8(11):1159-1162.

[8] Patel SR, Vadhan-Raj S, Burgess MA, et al. Results of two consecutive trials of dose-intensive chemotherapy with doxorubicin and ifosfamide in patients with sarcomas. American Journal of Clinical Oncology: Cancer Clinical Trials. 1998; 21(3):317-321.

[9] De Pas T, De Braud F, Orlando L, et al. High-dose ifosfamide plus adriamycin in the treatment of adult advanced soft tissue sarcomas: is it feasible? Annals of Oncology. 1998;9(8):917-919.

[10] Reichardt R, Tilgner J, Hohenberger P, Dörken B. Doseintensive chemotherapy with ifosfamide, epirubicin, and filgrastim for adult patients with metastatic or locally advanced soft tissue sarcoma: a phase II study. Journal of Clinical Oncology. 1998;16(4):1438-1443.

[11] Palumbo R, Toma S. Feasibility and compliance of epirubicin plus ambulatory continuous infusion ifosfamide at escalating doses in advanced soft tissue sarcomas: a phase I study. European Journal of Cancer Part A: General Topics. 1995; 31(9):1552-1553.

[12] Buesa JM, López-Pousa A, Martín J, et al. Phase II trial of firstline high-dose ifosfamide in advanced soft tissue sarcomas of the adult: a study of the Spanish Group for Research on Sarcomas (GEIS). Annals of Oncology. 1998;9(8):871-876.

[13] Miller AB, Hoogstraten B, Staquet M, Winkler A. Reporting results of cancer treatment. Cancer. 1981;47(1):207-214.

[14] Gehan EA. The determination of the number of patients required in a preliminary and follow-up trial of a new chemotherapeutic agent. Journal of Chronic Diseases. 1961;13: 346-353.

[15] Leyvraz S, Bacchi M, Cerny T, et al. Phase I multicenter study of combined high-dose ifosfamide and doxorubicin in the treatment of advanced sarcomas. Annals of Oncology. 1998;9(8):877-884.

[16] Worden FP, Taylor JMG, Biermann JS, et al. Randomized phase II evaluation of $6 \mathrm{~g} / \mathrm{m}^{2}$ of ifosfamide plus doxorubicin and granulocyte colony-stimulating factor (G-CSF) compared with $12 \mathrm{~g} / \mathrm{m}^{2}$ of ifosfamide plus doxorubicin and G-CSF in the treatment of poor-prognosis soft tissue sarcoma. Journal of Clinical Oncology. 2005;23(1):105-112.

[17] Frustaci S, Buonadonna A, Galligioni E, et al. Increasing 4' epidoxorubicin and fixed ifosfamide doses plus granulocytemacrophage colony-stimulating factor in advanced soft tissue sarcomas: a pilot study. Journal of Clinical Oncology. 1997; 15(4):1418-1426.

[18] Palumbo R, Neumaier C, Cosso M, et al. Dose-intensive firstline chemotherapy with epirubicin and continuous infusion ifosfamide in adult patients with advanced soft tissue sarcomas: a phase II study. European Journal of Cancer. 1999; 35(1):66-72.

[19] Maurel J, Fra J, López-Pousa A, et al. Sequential dose-dense doxorubicin and ifosfamide for advanced soft tissue sarcomas: a phase II trial by the Spanish Group for Research on Sarcomas (GEIS). Cancer. 2004;100(7):1498-1506.

[20] De Pas T, Curigliano G, Masci G, et al. Phase I study of twelveday prolonged infusion of high-dose ifosfamide and doxorubicin as first-line chemotherapy in adult patients with advanced soft tissue sarcomas. Annals of Oncology. 2002;13(1): 161-166. 
[21] Maurel J, Buesa JM, López-Pousa A, et al. Salvage surgical resection after high-dose ifosfamide (HDIF) based regimens in advanced soft tissue sarcoma (ASTS): a potential positive selection bias. A study of the Spanish Group for Research on Sarcomas (GEIS). Journal of Surgical Oncology. 2004;88(1):4449.

[22] Di Maio M, Gridelli C, Gallo C, et al. Chemotherapy-induced neutropenia and treatment efficacy in advanced non-smallcell lung cancer: a pooled analysis of three randomised trials. Lancet Oncology. 2005;6(9):669-677.

[23] Gurney H. I don't underdose my patients... do I? Lancet Oncology. 2005;6(9):637-638. 


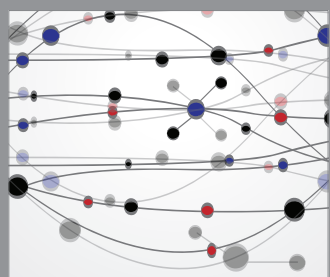

The Scientific World Journal
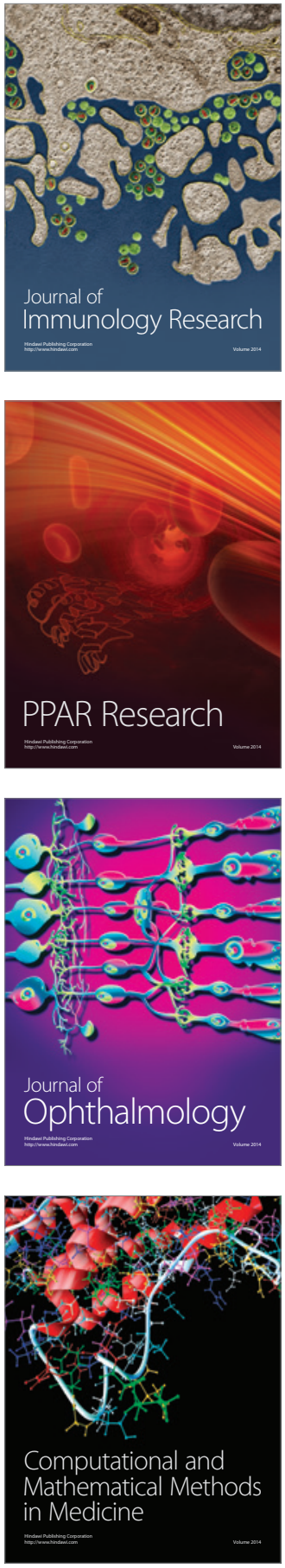

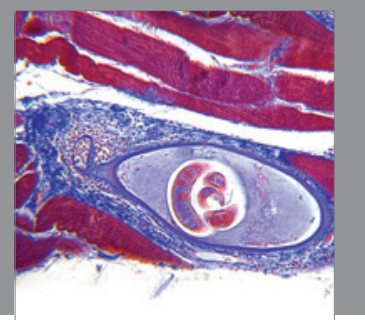

Gastroenterology

Research and Practice
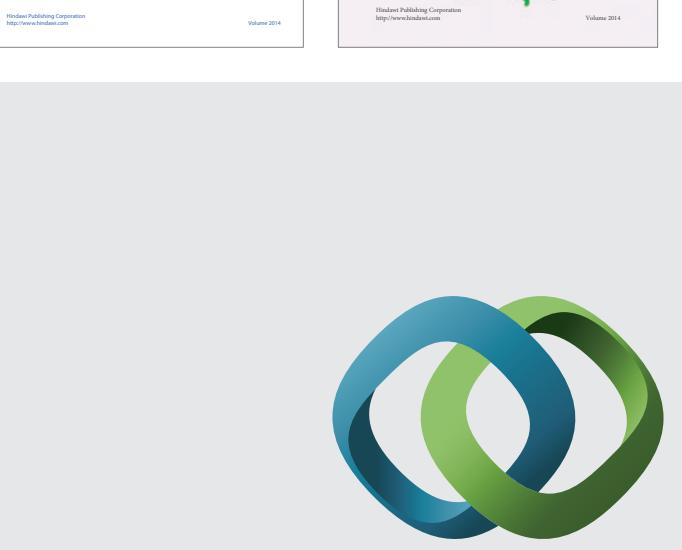

\section{Hindawi}

Submit your manuscripts at

http://www.hindawi.com
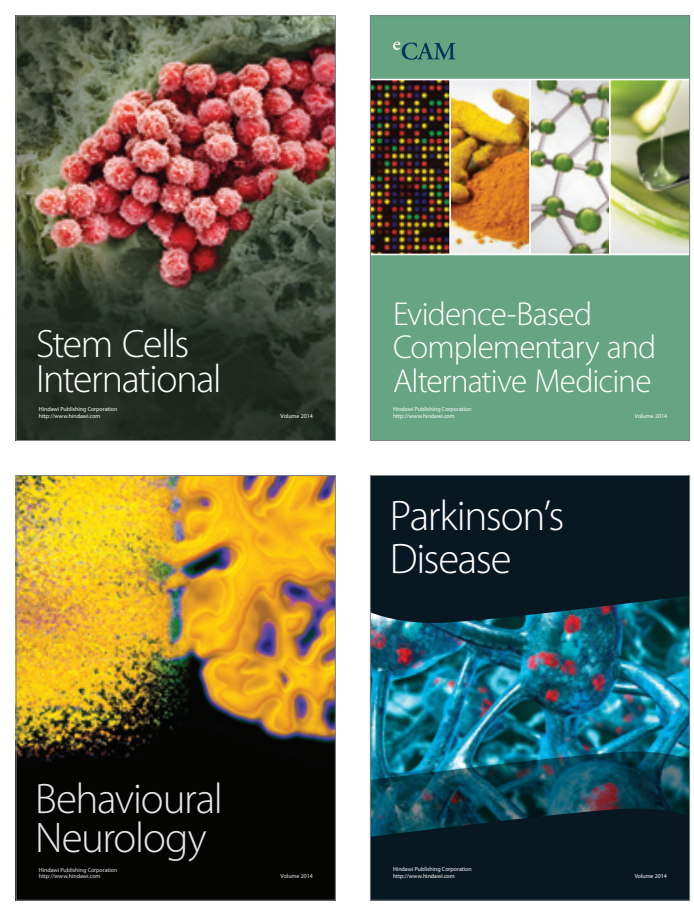

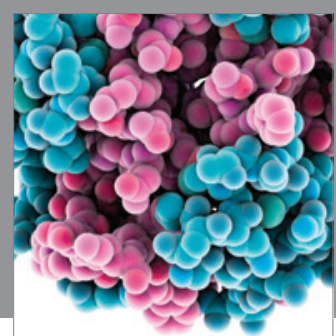

Journal of
Diabetes Research

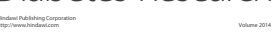

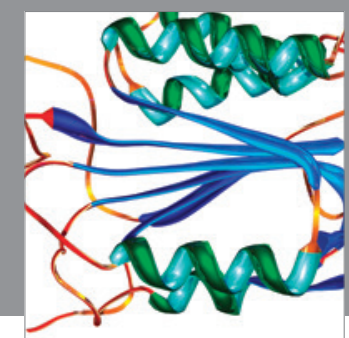

Disease Markers
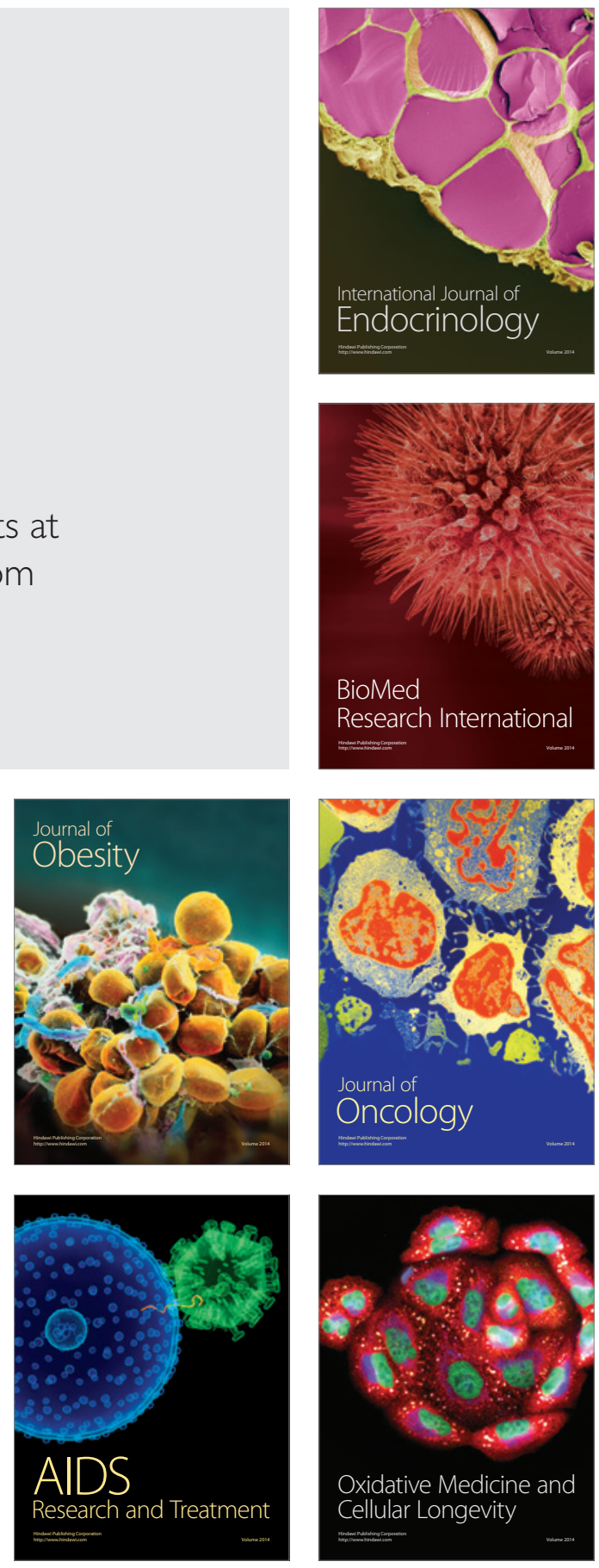